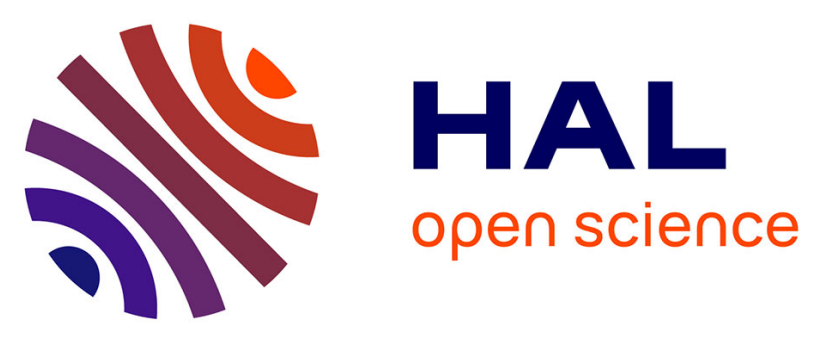

\title{
Recherches sur la résistance des sols aux maladies. IX. - Dynamique des populations du Fusarium spp. et de Fusarium oxysporum f. sp. melonis dans un sol résistant et dans un sol sensible aux fusarioses vasculaires
}

Claude Alabouvette, Yvonne Couteaudier, Jean Louvet, Marie-Louise Soulas

\section{To cite this version:}

Claude Alabouvette, Yvonne Couteaudier, Jean Louvet, Marie-Louise Soulas. Recherches sur la résistance des sols aux maladies. IX. - Dynamique des populations du Fusarium spp. et de Fusarium oxysporum f. sp. melonis dans un sol résistant et dans un sol sensible aux fusarioses vasculaires. Agronomie, 1984, 4 (8), pp.729-733. hal-00884690

\section{HAL Id: hal-00884690 \\ https://hal.science/hal-00884690}

Submitted on 1 Jan 1984

HAL is a multi-disciplinary open access archive for the deposit and dissemination of scientific research documents, whether they are published or not. The documents may come from teaching and research institutions in France or abroad, or from public or private research centers.
L'archive ouverte pluridisciplinaire HAL, est destinée au dépôt et à la diffusion de documents scientifiques de niveau recherche, publiés ou non, émanant des établissements d'enseignement et de recherche français ou étrangers, des laboratoires publics ou privés. 


\section{Recherches sur la résistance des sols aux maladies. IX. - Dynamique des populations du Fusarium spp. et de Fusarium oxysporum f. sp. melonis dans un sol résistant et dans un sol sensible aux fusarioses vasculaires}

Claude ALABOUVETTE, Yvonne COUTEAUDIER \& Jean LOUVET avec la collaboration technique de Marie-Louise SOULAS

I.N.R.A., Station de Recherches sur la Flore pathogène dans le Sol, 17, rue Sully, F 21034 Dijon Cedex

L'emploi d'un mutant de Fusarium oxysporum f. sp. melonis résistant au bénomyl nous a permis de suivre parallèlement l'évolution de la population de l'agent pathogène et celle de la population totale des Fusarium spp. dans un sol résistant et un sol sensible aux fusarioses vasculaires (sols de (hâteaurenard et d'Ouroux). Nous avons ainsi pu démontrer que la dynamique de l'inoculum de $F$. o. f. sp. melonis est identique en terre résistante et en terre sensible. Introduit aux mêmes doses initiales de 100 et 1000 propagules $/ \mathrm{g}$ de sol, l'agent pathogène s'établit dans les sols à un niveau 10 fois plus faible mais comparable en terre résistante et en terre sensible. En absence de plante (fig. 1) la population de l'agent pathogène évolue peu au cours du temps et il est toujours possible de mettre en évidence $F$. $O$. f. sp. melonis plus d'un an après son introduction, aussi bien dans le sol résistant que dans le sol sensible. En présence d'une plante sensible, la population de l'agent pathogène tend à augmenter en terre sensible en relation avec l'évolution de la maladie (fig. 3, tabl. 1).

Les résultats acquis confirment nos observations antérieures montrant l'importance numérique des Fusarium spp. sauvages dans le sol résistant, où ils sont 10 fois plus abondants qu'en terre sensible. L'agent pathogène s'installant à des niveaux comparables dans les 2 sols, la valeur du rapport : densité de la population de $F$. oxysporum pathogène/densité de la population fusarienne totale est 10 fois plus faible en terre résistante qu'en terre sensible (tabl. 2). La valeur de ce rapport serait en corrélation avec le niveau de réceptivité des sols aux fusarioses vasculaires et déterminerait la gravité de la maladie.

Mots clés additionnels : Champignon pathogène, inoculum, rapport de populations, réceptivité du sol, melon. and $\mathrm{F}$. oxysporum $f$. sp. melonis in a wilt-suppressive and a wilt-conducive soil.

The development of the Fusarium population pathogenic to muskmelons and of the total Fusarium population was studied in a wilt-conducive soil and a wilt-suppressive soil, using a benomyl-resistant mutant of $F$. $o$. f. $\mathrm{sp}$. melonis. The population dynamics (i.e. the fluctuation of inoculum density with time) of the pathogenic strain were generally about the same in the 2 soils.

When introduced into samples of each soil, at the same initial densities of 100 and 1000 propagules/g soil, the populations stabilized at about 10 and 100 propagules/g soil in both the conducive and suppressive soils. In the absence of plants, the density of the pathogen population changed little with time, and the pathogen was still detected in both soils more than one year later (fig. 1).

When susceptible host-plants were grown, the density of the pathogen population changed little in the suppressive soil, but increased in the conducive soil, the amount of increase being proportional to disease severity (fig. 3, table 1). The population of native non pathogenic Fusarium spp. was 10 times greater in the suppressive than in the conducive soil. Because the pathogenic population stabilized at comparable levels in the 2 soils, the ratio population density of pathogenic $F$. oxysporum/total population density of Fusarium spp. in the suppressive soil was only $1 / 10$ of that in conducive soil (table 2 ). In correlation with the level of receptivity of the soil to Fusarium wilts, it could determine the disease incidence.

Additional key words : Pathogenic fungi, inoculum, population ratio, soil receptivity, muskmelon. 


\section{INTRODUCTION}

Afin de déterminer le niveau de réceptivité des sols aux fusarioses vasculaires, nous avons mis au point une méthode qui consiste à apporter aux sols des quantités croissantes d'un inoculum standard et à enregistrer l'évolution corrélative de la mortalité des plantes (ALABOUVETTE et al., 1982). Les sols dans lesquels la maladie ne se manifeste pas, ou faiblement, sont qualifiés de sols résistants. Ce protocole expérimental basé sur un apport d'inoculum, invite à étudier l'installation puis l'évolution de l'agent pathogène dans les sols résistants. En effet, certains auteurs comme BAKER \& COOK (1974) distinguent 2 catégories de sols résistants : ceux dans lesquels l'agent pathogène ne peut pas s'installer et ceux dans lesquels il s'installe mais ne produit pas la maladie. Ces 2 types de sols résistants recouvrent des mécanismes microbiologiques très différents et il est donc intéressant de connaître l'évolution des populations de l'agent pathogène dans ces sols.

Dès les premiers travaux consacrés à l'étude des sols résistants de Châteaurenard, nous avons démontré que l'inoculum préalablement introduit peut encore y être décelé après 2 mois $1 / 2$ de culture et qu'il a conservé ses capacités infectieuses et pathogènes (LOUVET et al., 1976). L'analyse du niveau global des populations de $F$. oxysporum semblait indiquer une multiplication de l'agent pathogène dans le sol sensible et une faible diminution de la densité d'inoculum dans le sol résistant (AlaBouvetTE et al., 1979). Mais les difficultés inhérentes à la détermination de la forme spéciale, par isolement et inoculation à la plante sensible de toutes les colonies de $F$. oxysporum, n'ont pas permis de suivre avec précision l'évolution de la population de l'agent pathogène ; il n'a pas été possible de confirmer la validité de ces premières observations.

L'obtention d'un mutant de $F$. $o$. f. sp. melonis résistant au bénomyl nous a permis d'aborder avec plus de précision l'étude comparative de la dynamique de l'inoculum. Dans ce but, nous avons analysé périodiquement le niveau global des populations de Fusarium spp. ainsi que la densité des populations de l'agent pathogène dans les sols préalablement infestés aux doses théoriques de 100 et 1000 propagules de $F$. o. f. sp. melonis par g de sol. Afin de bien distinguer l'influence des sols eux-mêmes de celle liée à la présence des plantes, nous avons réalisé parallèlement 2 types d'expérimentation : la dynamique de l'inoculum a été suivie, d'une part dans les sols maintenus humides en absence de culture et, d'autre part, dans les sols cultivés avec une variété de melon sensible ou résistante au pathotype de $F . o$. sp. melonis présent dans les sols.

\section{MATÉRIEL ET MÉTHODES}

Les caractéristiques agronomiques du sol résistant de Châteaurenard et du sol sensible d'Ouroux utilisés dans cette étude ont été publiés précédemment (ALABOUVETTE et al., 1980). Leur infestation aux doses de 100 et 1000 propagules par $\mathrm{g}$ de sol est réalisée avec un inoculum de $F . o$. f. sp. melonis, souche $\mathrm{C} 1$ résis- tante au bénomyl, selon le protocole décrit précédemment (AlabouvetTE et al., 1982).

\section{A. Conditions expérimentales}

Dans le cas des sols non cultivés, des échantillons d'une dizaine de $\mathrm{kg}$ sont répartis dans des bacs placés dans un local tempéré $\left(18-25^{\circ} \mathrm{C}\right)$. Après infestation et homogénéisation, l'humidité est réglée à $20 \mathrm{p} .100$ en terre résistante et à 10 p. 100 en terre sensible, ce qui correspond à un potentiel hydrique voisin de -1 bar.

Dans le cas des essais conduits avec plantation, les sols après infestation sont répartis dans des pots de 11 dans chacun desquels on repique une plantule de melon de la variété "Charentais ", sensible à la race 0 de $F$. o. f. sp. melonis souche $\mathrm{C} 1$, ou de la variété " Doublon », résistante. La culture est conduite en serre, dans des conditions favorables à l'expression de la maladie $\left(18-22{ }^{\circ} \mathrm{C}\right)$. Chaque traitement porte sur 15 plantes.

Les échantillons de sol destinés aux analyses sont constitués par des prélèvements dans 2 pots choisis au hasard pour chaque traitement.

\section{B. Analyse des populations fusariennes}

Après prélèvement, les échantillons de sol de $300 \mathrm{~g}$ environ sont mis à sécher dans une enceinte ventilée durant $60 \mathrm{~h}$, puis subissent un broyage et un tamisage selon la technique décrite par ROUXEL \& BOUHOT (1971). La fraction inférieure à $200 \mu \mathrm{m}$ est analysée sur le milieu proposé par KOMADA (1975) afin de dénombrer l'ensemble des Fusarium spp. et également sur le même milieu additionné de $5 \mu \mathrm{g} / \mathrm{ml}$ de bénomyl afin de dénombrer sélectivement l'agent pathogène résistant à ce fongicide. Le dénombrement des colonies est effectué après une semaine d'incubation à $22{ }^{\circ} \mathrm{C}$ et à la lumière du jour.

\section{RÉSULTATS}

Les résultats exprimés en nombre de propagules par $\mathrm{g}$ de sol sec sont présentés graphiquement : les dates d'analyse figurent en abscisse sur une échelle arithmétique et les densités d'inoculum figurent en ordonnée sur une échelle logarithmique. Chaque graphique présente, d'une part, le niveau des populations fusariennes totales déterminées dans les terres résistante et sensible non infestées et, d'autre part, les densités d'inoculum de $F$. $o$. f. sp. melonis analysées dans les terres infestées. Les résultats de l'expérience rapportée ici sont représentatifs de l'ensemble des résultats acquis au cours d'une expérimentation beaucoup plus importante, conduite sur une période de 3 années.

La figure 1 présente l'évolution de la densité des populations de Fusarium dans les sols maintenus humides, mais sans culture. Il apparaît que la population fusarienne totale est environ 10 fois plus importante dans le sol résistant que dans le sol sensible. Mais ces populations de niveau très différent présentent un comportement analogue : elles demeurent globalement stables tout au long de l'expérience. L'agent pathogène s'établit à des niveaux faibles, proportionnels aux doses d'apport et parfaitement comparables 
dans les 2 sols : les populations, très faibles lors de la $1^{\text {re }}$ analyse, augmentent entre la $6^{\mathrm{e}}$ et la $12^{\mathrm{e}}$ semaine puis diminuent à nouveau au-delà de la $25^{\mathrm{e}}$ semaine. Cependant, plus d'un an après son introduction à la dose la plus forte, $F$. o. f. sp. melonis est toujours présent. Les densités de population sont alors comparables dans le sol résistant et dans le sol sensible.

La figure 2 présente l'évolution de la densité des populations de Fusarium dans les sols cultivés avec une variété de melon résistante au pathotype de $F$. $o$. f. sp. melonis ayant servi à l'infestation. L'évolution des populations est globalement semblable à celle observée en sols non cultivés.

La figure 3 présente l'évolution de la densité des populations de Fusarium dans les sols cultivés avec une variété de melon sensible. Dès la $1^{\text {re }}$ date d'analyse, 5 semaines après l'infestation, les populations de l'agent pathogène s'établissent à des niveaux comparables en terre résistante et en terre sensible ; ils sont proportionnels aux doses d'apport initiales et environ 10 fois plus faibles que celles-ci. En terre résistante, ces populations évoluent peu au cours du temps. En terre sensible, l'évolution des populations de l'agent pathogène semble en rapport avec la gravité de la maladie présentée dans le tableau 1. Pour la dose d'infestation la plus forte, la population de l'agent pathogène augmente nettement entre la $10^{\mathrm{e}}$ et la $15^{\mathrm{e}}$ semaine alors que toutes les plantes présentent

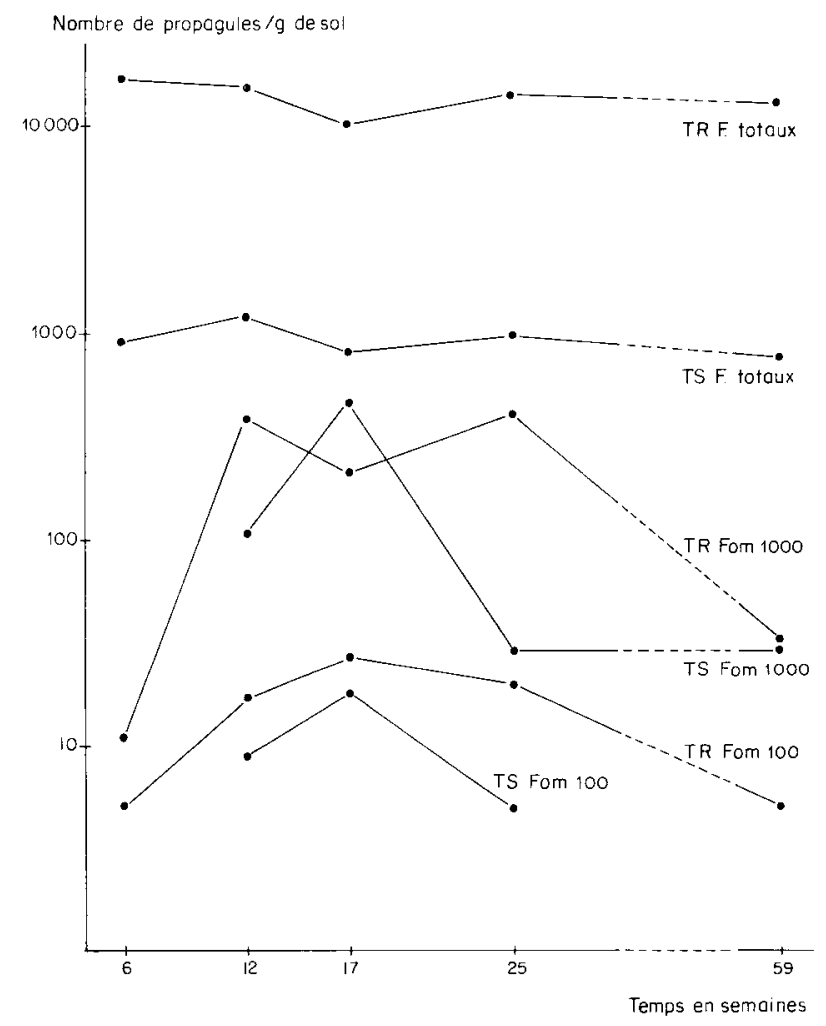

Figure 1

Evolution au cours du temps de la densité des populations de Fusarium spp. (F. totaux) et de F. o. f. sp. melonis (Fom) dans les terres résistantes (TR) et sensible (TS) non (ultivées. (log du nombre de propagules $/ g$ de sol-temps en semaines).

Fom 100, Fom 1000 ; infestation initiale du sol avec respectivement 100 et 1000 propagules de Fom par $g$ de sol.

Development of populations of Fusarium spp. (F. totaux) and F. o. f. $s p$. melonis (Fom) in non-cultivated suppressive (TR) and conducive (TS) soils (log number of propagules/g soil-time in weeks). Fom 100, Fom 1000 : initial introduction of 100 and 1000 propagules of Fom/g soil. déjà des symptômes caractéristiques. Pour la dose d'infestation la plus faible, la maladie évolue plus lentement et la densité d'inoculum croît lentement entre la $10^{\mathrm{e}}$ et la $15^{\mathrm{e}}$ semaine, plus nettement entre la $15^{\mathrm{e}}$ et la $20^{\mathrm{e}}$ semaine. Durant cette dernière période, les plantes ayant été arrachées, on peut semble-t-il attribuer cette augmentation de la densité d'inoculum à un développement de l'agent pathogène sur les restes de racines préalablement infectées. La figure 3 indique également que la population totale de Fusarium augmente faiblement et de façon parallèle dans la terre sensible au cours de cette expérience.

Ces résultats montrent une nouvelle fois que la population totale de Fusarium est beaucoup plus élevée dans le sol résistant que dans le sol sensible. Etant donné que la population de l'agent pathogène s'établit dans ces 2 types de sol à un niveau comparable, il en résulte que l'importance relative de la population pathogène au sein de la population fusarienne totale est beaucoup plus faible en terre résistante qu'en terre sensible. Le tableau 2 indique le pourcentage de l'agent pathogène par rapport aux populations fusariennes totales. Malgré des fluctuations importantes

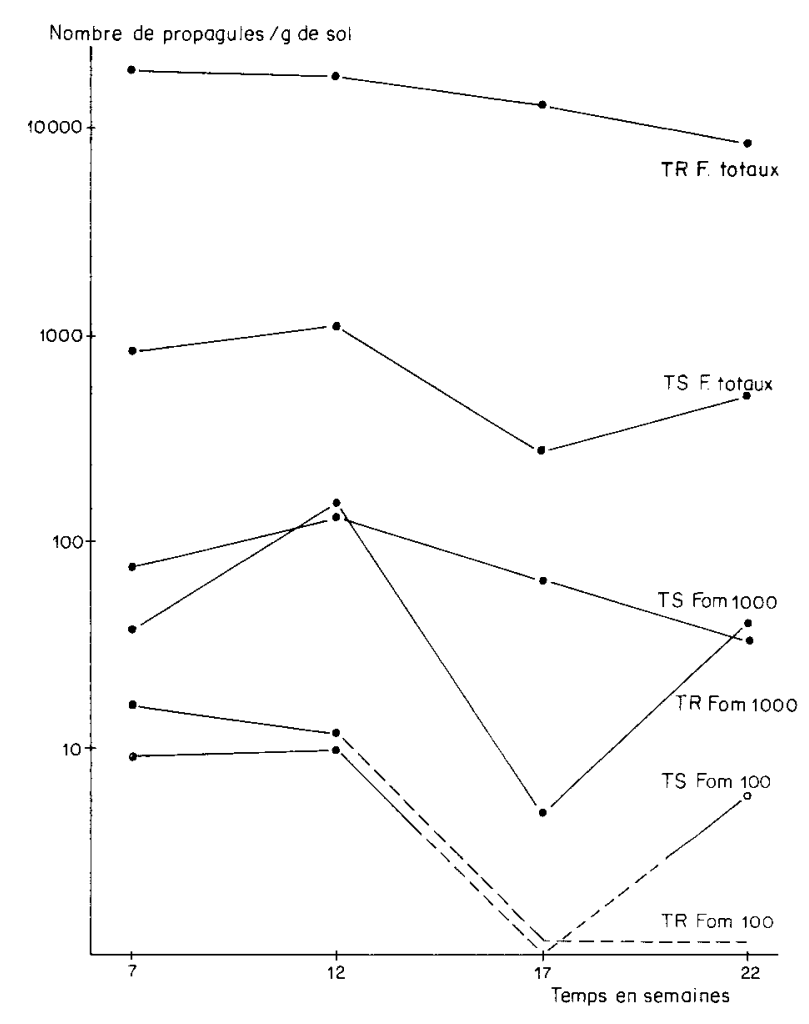

Figure 2

Evolution au cours du temps de la densité des populations de Fusarium spp. (F. totaux) et de F. o. $f$. sp. melonis (Fom) dans les terres résistantes (TR) et sensible (TS) en présence de melons (var. "Doublon ») résistants (log du nombre de propagules/g de sol-temps en semaines).

Fom 100, Fom 1000 : infestation initiale du sol respectivement 100 et 1000 propagules de Fom par $\mathrm{g}$ de sol.

(Les lignes discontinues correspondent à des dénombrements trop faibles pour être pris en compte.)

Development of populations of Fusarium spp. (F. totaux) and F. o. f. sp. melonis (Fom) in suppressive (TR) and conducive (TS) soils cultivated with resistant muskmelons (cv. 'Doublon') (log number of propagules/g soil-time in weeks).

Fom 100, Fom 1000 : initial introduction of 100 and 1000 propagules of Fom/g soil.

(The dotted lines show numbers too low to be taken into account.) 


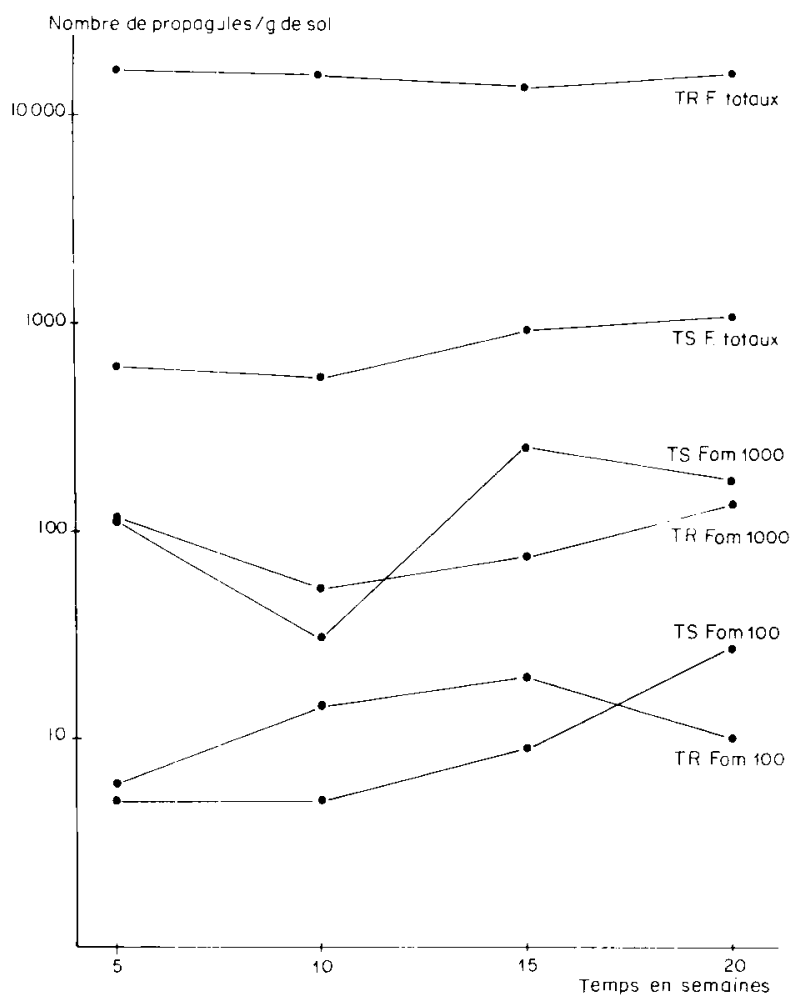

Figure 3

Evolution au cours du temps de la densité des populations de Fusarium spp. (F. totaux) et de F. o. f. sp. melonis (Fom) dans les terres résistante (TR) et sensible (TS) en présence de melons (var. "Charentais ») sensibles (log du nombre de propagules/g de sol-temps en semaines).

Fom 100, Fom 1000 : infestation initiale du sol avec respectivement 100 et 1000 propagules de Fom par $g$ de sol.

Development of populations of Fusarium spp. (F. totaux) and F. o. $f$. sp. melonis (Fom) in suppressive (TR) and conducive (TS) soils cultivated with susceptible muskmelon ( $c v$. 'Charentois') (log number of propagules' $g$ soil-time in weeks).

Fom 100, Fom 1000 : initial introduction of 100 and 1000 propagules of Fom/g soil.

d'une date d'analyse à l'autre, il apparaît que les pourcentages calculés sont toujours beaucoup plus faibles en sol résistant. Ainsi, l'apport de l'agent pathogène à la même concentration se traduit-il par une situa-

\section{TABLEAU 1}

Pourcentage de melons de la variété "Charentais " présentant des symptômes de fusariose après 10 et 15 semaines de culture en terres résistante et sensible infestées avec 100 et 1000 propagules de $\mathrm{F}$. 0 . $f$. $s p$. melonis par g (15 plantes/traitement).

Percentage of wilted plants at 10 and 15 weeks after planting in conducive and suppressive soils infested initially with 100 and 1000 propagules per $g$ of soil (15 plants/treatment).

\begin{tabular}{ccccc} 
& \multicolumn{2}{c}{ Terre résistante } & \multicolumn{2}{c}{ Terre sensible } \\
\hline $\begin{array}{c}\text { Doses } \\
\text { d'inoculum }\end{array}$ & 10 & 15 & 10 & 15 \\
\hline 0 & semaines & semaines & semaines & semaines \\
\hline 100 & 0 & 0 & 0 & 0 \\
\hline 1000 & 14 & 54 & 100 & 100 \\
\hline \hline
\end{tabular}

tion très différente: dans le sol résistant, l'agent pathogène ne représente qu'une très faible proportion de la population de Fusarium spp. alors qu'en sol sensible infesté à la dose la plus forte il constitue environ 10 à 18 p. 100 de cette population totale.

\section{DISCUSSION}

Les résultats présentés, confirmés par une expérience similaire, indiquent que la dynamique de la population de $F$. oxysporum pathogène en absence de plante sensible n'est pas fondamentalement différente dans le sol résistant et dans le sol sensible. L'agent pathogène y est encore présent plus de 14 mois après son introduction. Sa population s'établit puis se maintient à des niveaux qui sont fonction de la dose initiale d'infestation et non du type de sol. Ceci infirme donc l'hypothèse (ALABOUVETTE et al., 1979) selon laquelle la population de l'agent pathogène s'établirait à un niveau constant dans chaque sol, indépendamment de la dose d'apport initiale. Selon la terminologie de COOK \& BAKER (1983), nous sommes réellement en

TABLEAU 2

Pourcentage de la population de l'agent pathogène dans l'ensemble des populations fusariennes, calculé dans les analyses successives.

Percentage of the pathogenic F. o. f. sp. melonis in the total populations of Fusarium spp. in suppressive and conducive soils infested initially with 100 and 1000 propagules of F. o. $f . s p$. melonis per $g$ of soil, then incubated with or without susceptible plants.

Terre résistante

Terre sensible

\begin{tabular}{|c|c|c|c|c|c|c|c|c|c|c|c|}
\hline & \multirow{2}{*}{$\begin{array}{l}\text { Dose d'apport } \\
\text { de } F \text {.o. } \\
\text { f. sp. melonis }\end{array}$} & \multicolumn{4}{|c|}{ Analyses successives } & \multirow{2}{*}{ Moyenne } & \multicolumn{4}{|c|}{ Analyses successives } & \multirow{2}{*}{ Moyenne } \\
\hline & & 1 & 2 & 3 & 4 & & 1 & 2 & 3 & 4 & \\
\hline \multirow{2}{*}{$\begin{array}{c}\text { Terres } \\
\text { non } \\
\text { cultivées }\end{array}$} & 100 & 0,03 & 0,15 & 0,21 & 0,13 & 0,13 & 0 & 0,86 & 2,15 & 0,38 & 0,85 \\
\hline & 1000 & 0,07 & 2,33 & 2,27 & 2,61 & 1,82 & 0 & 9,11 & 26,7 & 2,85 & 9,67 \\
\hline \multirow{2}{*}{$\begin{array}{c}\text { Culture } \\
\text { de la variété } \\
\text { «Doublon » }\end{array}$} & 100 & 0,11 & 0,07 & 0 & 0 & 0,05 & 2,47 & 2,56 & 0 & 0 & 1,26 \\
\hline & 1000 & 0,40 & 0,84 & 0,49 & 0,39 & 0,54 & 8,69 & 21,3 & 1,78 & 7,18 & 9,74 \\
\hline \multirow{2}{*}{$\begin{array}{c}\text { Culture } \\
\text { de la variété } \\
\text { «Charentais }\end{array}$} & 100 & 0,09 & 0,10 & 0,15 & 0,06 & 0,10 & 0,94 & 0,48 & 0,76 & 2,57 & 1,19 \\
\hline & 1000 & 0,95 & 0,43 & 0,46 & 0,75 & 0,65 & 13,1 & 3,86 & 19,1 & 34,7 & 17,7 \\
\hline
\end{tabular}


présence d'un sol résistant à la maladie (disease suppressive soil) et non pas d'un sol résistant à l'agent pathogène (pathogen suppressive soil). Cette conclusion est importante car elle montre que, pour un sol donné, capacité d'accueil de l'agent pathogène et niveau de réceptivité à la maladie peuvent être 2 caractéristiques indépendantes.

Par ailleurs cette étude montre l'influence de la culture sur le niveau des populations de l'agent pathogène. En présence de plantes sensibles, le niveau de la population pathogène reste pratiquement stable en sol résistant alors qu'il tend à augmenter en terre sensible au-delà de la $10^{\mathrm{e}}$ semaine de culture, c'est-à-dire après apparition des symptômes. Il semble donc que l'on puisse attribuer cette augmentation à une multiplication de l'agent pathogène au niveau des racines des plantes infectées. Cette interprétation est confirmée par le fait qu'en présence de plantes résistantes à la maladie, la population de l'agent pathogène évolue de façon similaire en sol résistant et en sol sensible. WENSLEY \& MAC KEEN (1963), étudiant la dynamique des populations de $F$. oxysporum dans des sols résistant et sensible naturellement infestés par $F$. $o$. f. sp. melonis, ont également montré que la densité d'inoculum s'établit à un niveau identique dans les sols euxmêmes et qu'elle est plus élevée à proximité immédiate des plantes malades.

Les nombreuses analyses réalisées au cours de cette expérimentation confirment nos observations antérieures (ROUXEL et al., 1977) concernant l'abondance exceptionnelle des Fusarium spp. dans le sol résistant. WENSLEY \& MAC KEEN (1963) comme SMITH \& SNYDER (1971) ont déjà attiré l'attention sur le niveau élevé des populations de $F$. oxysporum non pathogènes dans les terres résistantes. Globalement, la population fusarienne totale du sol de Châteaurenard est 10 fois plus importante que celle du sol d'Ouroux. Etant donné que l'agent pathogène s'installe à un niveau sensiblement identique dans les 2 sols, le rapport densité de la population du $F$. oxysporum pathogène

densité de la population fusarienne totale

est donc environ 10 fois plus faible dans le sol résistant que dans le sol sensible. WENSLEY \& MAC KEEN (1963) estiment que le rapport entre la densité des $F$. oxysporum pathogènes et la densité totale des $F$. oxysporum décrit mieux l'état d'infestation d'un sol que la seule indication de la densité d'inoculum. Pour notre part, nous pensons que le niveau de réceptivité des sols qui conditionne l'expression de la maladie est en relation avec le rapport donné ci-dessus qui tient compte de la population fusarienne totale. Nos observations antérieures ont montré le rôle indispensable des Fusarium sauvages dans les mécanismes de la résistance des sols de Châteaurenard (ROUXEL et al., 1979). Une compétition intragénérique s'exercerait aussi bien dans la terre sensible que dans la terre résistante, mais elle serait d'autant plus défavorable à l'expression des $F$. oxysporum pathogènes qu'ils sont en plus faible proportion parmi la flore fusarienne du sol. Cette notion générale d'antagonisme entre champignons voisins tant du point de vue systématique qu'écologique a déjà été discutée par plusieurs auteurs et en particulier par DEACON (1976) dans le cas du piétin des céréales.

Nous formulons l'hypothèse selon laquelle les $F$. oxysporum pathogènes seraient soumis dans le sol résistant de Châteaurenard à une compétition telle que leur développement en direction de la racine serait inhibé. En effet, avant de pouvoir exercer leur pouvoir pathogène, les $F$. oxysporum doivent nécessairement parcourir une certaine distance dans le sol. C'est au cours de cette courte phase de développement saprophytique, se déroulant à proximité immédiate des racines, qu'interviendraient les mécanismes de compétition qui conditionnent le niveau de réceptivité des sols aux fusarioses vasculaires.

\section{Reçu le 6 janvier 1984.} Accepté le 9 avril 1984.

\section{RÉFÉRENCES BIBLIOGRAPHIQUES}

\begin{abstract}
Alabouvette C., 1983. La réceptivité des sols aux Fusarioses vasculaires, rôle de la compétition nutritive entre microorganismes. Thèse Doct. ès-Sci. Nat. Univ. Nancy, $158 \mathrm{p}$.

Alabouvette C., Rouxel F., Louvet J., 1979. Characteristics of Fusarium wilt suppressive soils and prospects for their utilization in biological control, 165-182, In Schippers B. \& Gams W., SoilBorne Plant Pathogens. Academic Press, 686 p.

Alabouvette C., Rouxel F., Louvet J., 1980. Recherches sur la résistance des sols aux maladies. VII. Etude comparative de la germination des chlamydospores de Fusarium oxysporum et Fusarium solani au contact des sols résistant et sensible aux fusarioses vasculaires. Ann. Phytopathol, 12 (1), 21-30.
\end{abstract}

Alabouvette C., Couteaudier Y., Louvet J., 1982. Comparaison de la réceptivité de différents sols et substrats de culture aux fusarioses vasculaires. Agronomie, 2 (1), 1-6.

Baker K. F., Cook R. J., 1974. Biological control of plant pathogens. Am. Phytopathol. Soc., St Paul MN, USA, 433 p.

Cook R. J., Baker K. F., 1983. The nature and practice of biological control of plant pathogens. Am. Phytopathol. Soc., St Paul MN, USA, 539 p.

Deacon J. W., 1976. Biological control of take-all fungus, Gaeumannomyces graminis by Phialophora radicicola and similar fungi. Soil Biol. Biochem., 8, 275-283.

Komada H., 1975. Development of a selective medium for quantita- tive isolation of Fusarium oxysportum from natural soil. Rev. Plant Protec. Res., 8, 114-125.

Louvet J., Rouxel F., Alabouvette C., 1976. Recherches sur la résistance des sols aux maladies. I. Mise en évidence de la nature microbiologique de la résistance d'un sol au développement de la fusariose vasculaire du melon. Ann. Phytopathol., 8, 425-436.

Rouxel F., Alabouvette C., Louvet J., 1977. Recherches sur la résistance des sols aux maladies. II. Incidence de traitements thermiques sur la résistance microbiologique d'un sol à la fusariose vasculaire du melon. Ann. Phytopathol., 9, 183-192.

Rouxel F., Alabouvette C., Louvet J., 1979. Recherches sur la résistance des sols aux maladies. IV. Mise en évidence du rôle des Fusarium autochtones dans la résistance d'un sol à la fusariose vasculaire du melon. Ann. Phytopathol., 11, 199-207.

Rouxel F., Bouhot D., 1971. Recherches sur l'écologie des champignons parasites dans le sol. IV. Nouvelles mises au point concernant l'analyse sélective et quantitative des Fusarium oxysporum et Fusarium solani dans le sol. Ann. Phytopathol., 3, 171-188.

Smith N., Snyder W. C., 1971. Relationship of inoculum density and soil types to severity of Fusarium wilt of sweet potato. Phytopathology, 61, 1049-1051.

Wensley R. N., McKeen C. D., 1963. Populations of Fusarium oxyosporum f. sp. melonis and their relation to the wilt potential of two soils. Can. J. Microbiol., 9, 237-249. 